\title{
61 Bacterial Sepsis and Shock
}

\author{
Jeffrey Alten - Priya Prabhakaran
}

\section{Introduction}

Severe sepsis and septic shock caused by bacterial infections are important causes of mortality and morbidity among children worldwide. Severe sepsis has a mortality of about $4.2 \%$ ( $2.3 \%$ in previously healthy children and $7.8 \%$ in chronically ill children). The incidence is greatest in children less than 1 year of age and contributes to $50 \%$ of the mortality in this age group. Survival from severe sepsis has improved from earlier years, attributable to the discovery that early recognition, aggressive resuscitation, and timely treatment improve outcomes in infants and children with severe sepsis.

\section{Microbiology of Sepsis in Children}

The epidemiology of sepsis varies depending on the age of the child, immunization status, integrity of the immune system, and presence of comorbidities. The etiologies of invasive childhood bacterial infections have been significantly modified by widespread immunization against Hemophilus influenza type B (Hib), as well as pneumococcal serotypes. It is convenient to classify the most common bacterial infections based on age of the child. The organisms that should be considered in the septic neonate include Group B Streptococcus, Listeria monocytogenes, and Gram-negative enteric bacteria such as Escherichia coli, Klebsiella pneumoniae, Enterobacter species, and Pseudomonas aeruginosa. Staphylococci, particularly coagulase negative species, are becoming an important cause of sepsis in hospitalized neonates. Of note, herpes simplex virus can cause septic shock often indistinguishable from bacterial septic shock. It is not uncommon for neonates with severe herpes infections to have a concomitant bacteremia due to Gramnegative organisms, presumably caused by bacterial translocation from the intestines. In a recent large international severe sepsis therapy trial, Staphylococcus species were the most common cause of sepsis in children. In children under 1 year of age, community-acquired organisms such as Pneumococcus and Neisseria meningitides are frequent causes of sepsis. In the developing world, these two organisms, in addition to Salmonella species and Hemophilus influenza type $B$, are the most frequent causes of sepsis in infants. The most frequent pathogens remain the same in healthy school-aged children. In addition, sepsis without a clearly defined focus of infection such as meningococcemia becomes more common in this age group. Disseminated staphylococcal and streptococcal infections, often associated with a source in bone, joint, lung, or heart is a frequent cause of sepsis in children over 6 years of age. The incidence of methicillin resistance among Staphylococcus species has increased dramatically in developed countries.

Children with preexisting morbidities, such as congenital heart disease, genitourinary abnormalities, cancer, burns, sickle cell disease, severe malnutrition, and immune deficiencies, are at an increased risk of developing severe sepsis. In addition, neonates and other children that are hospitalized or who have had significant exposure to health care facilities are at increased risk of developing sepsis; importantly with infections caused by atypical organisms which may be resistant to the usual, first-line antibiotic therapy. Multidrug-resistant Gram-negative organisms have become increasingly prevalent among hospitalized children, especially in developing countries, and should influence the choice of initial antibiotic therapy. Knowledge of specific bacterial infections associated with underlying dysfunction in specific components of the immune system is essential. $(1$ Table 61.1 shows the common bacterial infections that are associated with specific pediatric conditions.

\section{Definition of Sepsis and Septic Shock}

The clinical spectrum of bacterial sepsis ranges from the early signs of bacterial invasion or toxin release in the body (such as tachycardia and fever) to circulatory collapse, multiple organ system failure, and death. To further classify this spectrum, consensus definitions for pediatric sepsis were developed by pediatric experts to help determine the severity of a child's illness and follow response to treatment. Each category represents an increase in severity of illness. 
Table 61.1

Specific organisms associated with sepsis in pediatric patients with comorbidities

\begin{tabular}{|l|l|}
\hline Condition & Organism \\
\hline $\begin{array}{l}\text { Sickle cell disease } \\
\text { (asplenia) }\end{array}$ & Pneumococcus, Salmonella \\
\hline Asplenia, polysplenia & Pneumococcus, Salmonella \\
\hline $\begin{array}{l}\text { Nephrotic syndrome } \\
\text { (complement deficiency) }\end{array}$ & Pneumococcus \\
\hline $\begin{array}{l}\text { Complement deficiency } \\
\text { (C6-9) }\end{array}$ & $\begin{array}{l}\text { Neisseria species } \\
\text { (meningococcus, gonococcus) }\end{array}$ \\
\hline Neutropenia & $\begin{array}{l}\text { Streptococcus mitis, } \\
\text { Streptococcus viridans, } \\
\text { Gram-negative }\end{array}$ \\
\hline AIDS & Pneumococcus \\
\hline Burns & Pseudomonas \\
\hline
\end{tabular}

Systemic inflammatory response syndrome (SIRS): An inflammatory state affecting the entire body that represents the body's immune response to an insult that may or may not be infection. The presence of two or more of the following criteria (one of which must be abnormal leukocyte count or temperature) identify patients with SIRS:

1. Core temperature (rectal, oral, bladder, esophageal) $>38.5^{\circ} \mathrm{C}$ of $<36^{\circ} \mathrm{C}$

2. Tachycardia $>2$ standard deviations above a child's normal-for-age heart rate, or for children $<1$ year, bradycardia $<10$ th percentile

3. Respiratory rate $>2$ standard deviations above child's normal for age

4. Leukocyte count either high or low for age or leukocyte differential with greater than $>10 \%$ immature neutrophil forms

Sepsis: Systemic inflammatory response syndrome (SIRS) due to suspected or proven infection.

Severe sepsis: Sepsis associated with either cardiovascular organ dysfunction, acute respiratory distress syndrome, or with any two or more other organ dysfunctions. The consensus definitions for organ dysfunction are the following:

1. Cardiovascular: Hypotension or need for vasoactive medicine to keep BP in the normal range, or two or more of the following: unexplained metabolic or lactic acidosis, oliguria, capillary refill $>5 \mathrm{~s}$

2. Respiratory: $\mathrm{PaO}_{2} / \mathrm{FiO}_{2}$ ratio $<300$ or $\mathrm{PaCO}_{2}>65$ Torr or $>0.5 \mathrm{FiO}_{2}$ to maintain saturations $>92 \%$ or need for mechanical ventilation
3. Neurologic: Glasgow Coma Score $\leq 11$ or acute deterioration in mental status

4. Hematologic: Platelet count $<80,000 / \mathrm{mm}^{3}$ or $50 \%$ decrease in platelet count or INR $>2$

5. Renal: Serum creatinine $\geq 2$ times upper limit for normal or twofold increase in baseline creatinine

6. Hepatic: Total bilirubin $\geq 4 \mathrm{mg} / \mathrm{dL}$ (not applicable for newborn) or ALT $>2$ times upper limit for age

Septic Shock: Sepsis with cardiovascular dysfunction despite administration of $\geq 40 \mathrm{~mL} / \mathrm{kg}$ isotonic fluid over $1 \mathrm{~h}$.

While these categorical definitions do not exactly define prognosis, morbidity and mortality likely increases along the continuum of SIRS to septic shock. Increasing number of organ dysfunction in children with severe sepsis increases the likelihood of mortality.

\section{Pathophysiology of Shock}

Shock can be defined as a condition of insufficient delivery of oxygen and nutrients to meet the metabolic demands of the body. Oxygen delivery $\left(\mathrm{DO}_{2}\right)$ is directly proportional to cardiac output and the oxygen content in arterial blood $\left(\mathrm{CaO}_{2}\right)$. Cardiac output depends on heart rate (HR) and stroke volume. Stroke volume is a function of preload, myocardial contractility, and afterload.

$$
\begin{gathered}
\mathrm{DO}_{2}=\mathrm{CO} \times \mathrm{CaO}_{2} \\
\mathrm{CO}=\mathrm{HR} \times \mathrm{SV} \\
\mathrm{CaO}_{2}=\left(\mathrm{Hb} \times 1.34 \times \mathrm{SaO}_{2}\right)+\left(0.003 \times \mathrm{PaO}_{2}\right)
\end{gathered}
$$

Oxygen delivery is an estimate of global oxygen availability, and may not be reflective of regional hypoperfusion and or ischemia. Severe sepsis induces a state of very high metabolic and oxygen demand. Inadequate $\mathrm{DO}_{2}$ evokes compensatory mechanisms in an attempt to restore the balance between oxygen demand and delivery. In the early stages of shock, abnormalities in oxygen transport balance are compensated via tachycardia and systemic vasoconstriction to improve organ perfusion and oxygen delivery. The ability of children to vasoconstrict causes systemic blood pressure to be preserved until later stages of shock when frank decompensation may occur. Children who have compensated shock frequently have normal or even elevated blood pressure, and as the shock state progresses, compensatory mechanisms are overwhelmed leading to hypotension and organ dysfunction. Irreversible shock follows when cellular dysfunction is so severe that death occurs even if 
cardiovascular function is restored to an adequate level. In order to achieve the best possible outcomes, shock should be diagnosed early and treated aggressively, before decompensation occurs.

\section{Early Recognition of Septic Shock}

\section{History}

A history focused on the child's activity level, intake and output, presence of fever, skin color or temperature, abnormal breathing, or rash is invaluable in making the diagnosis promptly, and may also aid in localizing the site of infection. History of risk factors for severe infection should be obtained, including immunization status, recent exposure to infectious illnesses, medication history (particularly recent use of systemic steroids), and presence of chronic illness.

\section{Clinical Findings and Physical Exam}

Rapid and repeated careful physical examination is essential to diagnose early shock and help localize the most likely source of the precipitating infection. Early recognition of shock requires a high index of suspicion, as only subtle changes in vital signs (i.e., mild tachycardia, mild tachypnea, or slight delayed capillary refill) may be the first signs of developing septic shock. In some forms of severe sepsis and septic shock, children present with "warm shock." Patients in warm shock have decreased systemic vascular resistance (SVR) with relative hypovolemia from vasodilatation and venous pooling of blood. There is low diastolic blood pressure, and wide pulse pressure with resultant clinical findings of "bounding pulses and warm extremities." Despite the potential reassurance of "adequate pulses," the oxygen delivery in these patients is not adequate, thus they are in shock. Children (especially infants and neonates) will more commonly present with "cold shock." These patients have elevated SVR, with a narrow pulse pressure, cool mottled extremities, and reduced peripheral pulses. There will often be tachypnea as a compensation for metabolic acidosis, which may develop into increased work of breathing if ARDS develops or there is underlying pulmonary disease. The child will frequently have fever and a "toxic" or ill appearance. Of note, neonates and young infants may have septic shock without fever. As shock evolves, there will start to be clinical evidence of compromised organ function with worsening tachycardia, tachypnea, decreased urine output, and altered mental status. Decrease in systolic blood pressure is a late finding in septic shock, and represents decompensated stage of shock, which can rapidly be fatal. As the child progresses to irreversible shock, severe hypotension, lethargy, and respiratory failure are late and ominous findings.

\section{Diagnostic Testing}

Cultures should be obtained from all pertinent sites (CSF, blood, urine, genitourinary tract, skin lesions), if possible before initiation of antibiotics. Antibiotic therapy should not be delayed while waiting to obtain cultures, though. There are no specific laboratory tests to diagnose shock. Leukocyte count obtained on complete blood count may help support the diagnosis; while platelet count, liver function tests, coagulation studies, arterial blood gases, serum lactic acid, and serum creatinine will all help determine the degree of organ dysfunction. Serial blood gases and/or lactate levels may be useful in assessing the response to treatment. Bedside glucose measurement should be performed in all infants and neonates with septic shock, as their limited glycogen stores put them at risk for severe hypoglycemia. Ionized calcium should also be measured in all infants and neonates as it is often low in septic shock which may decrease myocardial contractility. Chest $\mathrm{x}$-ray should be performed to evaluate for pulmonary disease. Echocardiography to assess cardiac function should be performed, especially in children with evidence of compromised cardiac output in cold shock states.

\section{Management of Septic Shock}

Successful management and treatment of septic shock requires timely treatment of underlying cause and immediate reversal and stabilization of the hemodynamic derangements to optimize perfusion to compromised vital organs and limit the cellular damage before it becomes irreversible.

\section{Antibiotics}

Once the diagnosis of septic shock has been made, appropriate antibiotic therapy must be administered immediately, as ineffective or delayed antibiotic therapy is associated with increased mortality from septic shock. Antibiotics should not be delayed because of inability to obtain cultures. Broad-spectrum empiric antibiotics must 
be chosen based on the most likely organism implicated. This will depend upon the age of the child (see $\bullet$ Microbiology of Sepsis in Children above), location of infection, and the severity of illness. Knowledge of local sensitivity patterns is also very important in choosing antibiotics that are likely to be effective. In addition to initial broad-spectrum antibiotics to cover all of the most likely pathogens, some special considerations include: empiric treatment for MRSA should be considered in all children; Gram-negative and anaerobic coverage should be added for children with potential GI or GU source; immunocompromised, malnourished, and hospitalized children should receive initial empiric treatment with anti-pseudomonal antibiotics; and neonates should receive treatment for Listeria monocytogenes and herpes simplex virus. The antibiotic coverage should be tailored to the results of initial and repeat cultures as well as poor clinical response to initial empiric coverage.

\section{Initial Treatment of Shock}

The principle of treating shock is to reduce the imbalance between the oxygen demands of the tissues and oxygen delivery. While most therapy in early shock is directed toward increasing oxygen delivery, measures to curtail oxygen requirement are equally important and become more so as the shock state progresses. Fever, pain, and agitation all increase the metabolic rate and oxygen demand significantly. Children in shock frequently have increased oxygen demand by the muscles of respiration as they attempt to hyperventilate to compensate for metabolic acidosis. Aggressive control of fever, management of pain and sedation, and consideration of early intubation and mechanical ventilation to reduce oxygen demand of work of breathing, even in the absence of respiratory failure, are all very effective methods of decreasing oxygen requirements.

\section{Airway and Breathing}

As with all critically ill children, the first step of management is to support the airway and ensure adequate oxygenation and ventilation. Most children with septic shock will be tachypneic as compensation for their underlying metabolic acidosis, and should be given oxygen via face mask to help improve oxygen delivery to tissues. Children with septic shock can have as much as $40 \%$ of their cardiac output shunted to their respiratory muscles to support increased work of breathing. Mechanical ventilation can eliminate the excessive oxygen demand and help restore the balance between oxygen delivery and consumption by shunting cardiac output from the respiratory muscles to other vital organs. In addition, mechanical ventilation can help facilitate the use of sedation and improve fever control, which will also decrease the patient's oxygen demand and consumption. In the later stages of shock, there may be more recognized clinical indications for mechanical ventilation such as respiratory failure - in the presence of inadequate ventilation and/or oxygenation, obstructed airway, excessive work of breathing, or altered mental status, endotracheal intubation and mechanical ventilation should be instituted.

Caution should be exercised when performing endotracheal intubation in children with septic shock, as they are at risk for developing a sudden decrease in cardiac output from acute drop in preload. This may occur due to positive intrathoracic pressure caused by mechanical ventilation, inhibiting blood return back to the heart. Hypotension in this situation should be treated by aggressive fluid resuscitation (see below). The decrease in $\mathrm{CO}$ may also be due to the vasodilating properties of the medicines used to intubate the child with - which neutralize the compensatory endogenous vasoconstriction that is occurring in the child with septic shock. Medicines such as benzodiazepines, propofol, barbiturates, and opiates should be used with extreme caution for this reason. Some have suggested ketamine as the premedication of choice to facilitate mechanical ventilation due to its ability to release endogenous catecholamines with resultant increase in SVR and relative preservation of cardiovascular stability. Despites its ability to preserve hemodynamics, etomidate should not be used in children with septic shock because it suppresses the child's adrenal axis.

\section{Fluid Resuscitation}

Management of decreased oxygen delivery in shock begins with rapid intravenous administration of isotonic fluids. Vascular access should be established quickly, and placement of intraosseous catheter should be performed if IV access cannot be obtained within minutes. Children in septic shock have relative (due to vasodilation and capillary leak) and/or absolute hypovolemia. Early restoration of effective circulating volume is associated with improved outcomes. A $20 \mathrm{~mL} / \mathrm{kg}$ bolus of isotonic fluid such as $0.9 \%$ normal saline or Lactated Ringer's should be given as rapidly as possible (over $5 \mathrm{~min}$ ) for initial resuscitation. 
Reassessment of the child's end-organ perfusion and vital signs should be performed after this and any repeat fluid bolus to determine if the child needs continued fluid resuscitation. The following are physiologic goals that should guide the fluid resuscitation: normalization of heart rate and blood pressure, improvement in capillary refill and perfusion to the extremities, normal mental status, and urine output $>1 \mathrm{~mL} / \mathrm{kg} / \mathrm{h}$. Repeated $20 \mathrm{~mL} / \mathrm{kg}$ isotonic fluid boluses should be given until these physiologic goals are met. Patients with severe septic shock will often require $60-80 \mathrm{~mL} / \mathrm{kg}$ or more of isotonic fluid in the first hour of resuscitation. Ongoing capillary leak must be monitored and adequate volume should be administered to prevent further hypovolemia. Hypocalcemia and hypoglycemia should be evaluated for and corrected during the fluid resuscitation. A urinary catheter should be placed to accurately follow urine output continuously. Central venous access should be established in children who respond poorly to the first 3-4 boluses, and those with insufficient physiologic physiologic reserves at baseline. Central venous pressure (CVP) monitoring may be a helpful adjunct for monitoring. The development of hepatomegaly, gallop rhythm or new crackles on auscultation, in addition to a marked increase in CVP without concomitant improvement in hemodynamics are signs of possible fluid overload denoting that more fluid is unlikely to be beneficial. Children in septic shock frequently become progressively more edematous with ongoing resuscitation due to continuing capillary leak. The development of edema or anasarca should not deter continuing fluid administration, if it is indicated for restoration of hemodynamics and improvement in oxygen delivery. Serial physical examinations are crucial, and resuscitation should be modified based on the evolving clinical scenario.

The use of colloid for fluid resuscitation in children with septic shock is controversial. A large randomized controlled trial of crystalloids versus colloids ( $4 \%$ albu$\mathrm{min}$ ) for resuscitation of shock in adults showed that these were equivalent. In a subgroup analysis, patients with severe sepsis had a trend toward improved mortality when resuscitated with $4 \%$ albumin as opposed to normal saline. In children, a randomized controlled trial in children with dengue shock demonstrated earlier reversal of shock in patients who received $5 \%$ albumin, and a clinical practice paper on meninogococcal septic shock in children used 5\% albumin for resuscitation with mortality lower (5\%) than predicted. Crystalloids are less expensive and more readily available than albumin and should be used for initial resuscitation. Until there is more evidence on the safety and efficacy of albumin resuscitation in children with septic shock, it should be reserved for consideration in children that have already received over $60 \mathrm{~mL} / \mathrm{kg}$ fluid resuscitation with isotonic crystalloid.

Due to its crucial role in oxygen delivery, packed red blood cell transfusion should be given to keep the hemoglobin above $10 \mathrm{~g} / \mathrm{dL}$, and can be used as colloid during fluid resuscitation. This hemoglobin goal is part of an algorithm that has demonstrated decreased mortality in adult septic patients, and has been adopted as a pediatric goal in septic shock by expert consensus.

\section{Vasoactive Medications}

Children who remain in shock despite $60 \mathrm{~mL} / \mathrm{kg}$ of fluid resuscitation (fluid-refractory shock) should be started on vasoactive infusions preferably via a central venous line. Children with fluid-refractory shock should be treated in a pediatric intensive care unit with invasive monitoring devices such as central venous and arterial lines.

Low dose dopamine may be started in a peripheral IV while central venous access is obtained. Dopamine is the first-line vasoactive drug of choice in pediatric septic shock not responsive to fluid resuscitation alone. It may be started at $5 \mu \mathrm{g} / \mathrm{kg} / \mathrm{min}$ and rapidly titrated up to $10 \mu \mathrm{g} / \mathrm{kg} / \mathrm{min}$ for goals of improved perfusion and blood pressure. If physiologic hemodynamic goals are not met with dopamine and fluid alone (fluid-refractory, dopamine-resistant shock), epinephrine (titrated between 0.02 and $0.3 \mu \mathrm{g} / \mathrm{kg} / \mathrm{min}$ ) is indicated for children who are hypotensive and in "cold shock." Vasoconstrictors such as norepinephrine (titrated between 0.02 and $0.3 \mu \mathrm{g} / \mathrm{kg} / \mathrm{min}$ ) are the treatment of choice in children who are vasodilated and in warm shock. For children with warm shock and lowblood pressure resistant to norepinephrine, the addition of vasopressin has been used with some success.

Fluid resuscitation should continue while titrating up on vasoactive medications unless hepatomegaly or pulmonary edema develops. In addition to the physiologic goals mentioned above, titration of vasoactive medicines can also be guided by serial serum lactate levels and superior vena cava saturations, with goal $\geq 70 \%$. In children with femoral venous access, the trend in inferior vena cava saturations can be used to assess the adequacy of resuscitation. For children with normal blood pressure and cold shock, afterload reduction may be an important adjunct as these patients often have some level of myocardial dysfunction contributing to their shock; type III 
phosphodieterase inhibitors (Milrinone $0.5 \mathrm{mcg} / \mathrm{kg} / \mathrm{min}$ ) or vasodilators such as sodium nitroprusside and nitroglycerin may be beneficial.

\section{Hormonal Therapy}

Children with septic shock are at risk for adrenal insufficiency. The exact role of steroid replacement therapy, its safety, and the identification of pediatric septic patients that will benefit from steroid therapy is not well defined. At this point, there is no high-level evidence to support the use of steroids in children who are not at risk for adrenal insufficiency. The consensus of experts recommend patients with septic shock at risk for adrenal insufficiency (recent systemic steroid exposure, purpura fulminans, congenital adrenal hyperplasia, or history of hypothalamic/pituitary insufficiency) or patients that have catecholamine resistant shock should receive hydrocortisone therapy after a random serum cortisol is drawn, if possible. There has not been consensus on the exact dose of shock or stress dose steroids. The dose range has been reported from $2 \mathrm{mg} / \mathrm{kg} /$ day to $100 \mathrm{mg} / \mathrm{kg} /$ day. The common practice at our institution is to send a random serum cortisol, followed by $100 \mathrm{mg} / \mathrm{m}^{2}$ dose of hydrocortisone IV and treatment with $100 \mathrm{mg} / \mathrm{m}^{2} /$ day to be continued until the child's shock resolves if there is evidence of adrenal insufficiency (defined as dramatic hemodynamic improvement with steroid therapy or random cortisol less than $18 \mathrm{mg} / \mu \mathrm{L})$. Steroids are stopped if there is no evidence of adrenal insufficiency or clinical improvement in the child's hemodynamics after the initial hydrocortisone bolus.

Children with septic shock are at risk for hypocalcemia which may contribute to myocardial dysfunction. Serial serum-ionized calcium should be monitored and corrected to the normal value. Due to the risk of neurologic injury and myocardial dysfunction, hypoglycemia must be avoided. Neonates and infants are especially at risk due to their poor glycogen stores. Children in septic shock should receive continuous infusion of $10 \%$ dextrose in their intravenous fluids. Hyperglycemia may be a risk factor for mortality in children with septic shock. Some clinicians advocate treatment with insulin infusions to keep glucose in the normal range, although the validity of this therapy is still under investigation.

\section{Refractory Shock}

Patients with refractory shock despite all the above measures should have all reversible causes of refractory shock ruled out (pericardial tamopnade, tension pneumothorax, intrabdominal hypertension, adrenal insufficiency, and hypothyroidism) and then referred to a center capable of providing pediatric ECMO support.

\section{Summary}

Bacterial sepsis continues to be a major source of morbidity and mortality in children worldwide. Early recognition (fever, tachycardia, tachypnea, abnormal white blood cell count, and presence of organ dysfunction) and immediate aggressive treatment has been shown to improve outcomes in pediatric patients with bacterial sepsis. Within the first hour of treatment, in addition to the standard ABCs of resuscitation, the child should receive up to $60 \mathrm{~mL} / \mathrm{kg}$ fluid boluses and dopamine initiation if still in shock after $60 \mathrm{~mL} / \mathrm{kg}$ of fluid. Blood, urine, respiratory, and other indicated cultures should be sent, and appropriate broad-spectrum antibiotics should also be initiated within the first hour. Fever control and consideration of early intubation and mechanical ventilation to significantly decrease the body's oxygen demand should be given to any child in septic shock. Escalation and direction of further fluid and vasoactive medicine resuscitation is determined by repeated examination and clinical response to the first hour of therapy.

\section{References}

Akech S, Ledermann H, Maitland K (2010) Choice of fluids for resuscitation in children with severe infection and shock: systematic review. BMJ 341:c4416. doi:10.1136/bmj.c4416

Brierley J, Carcillo JA, Choong K, Cornell T, Decaen A, Deymann A, Doctor A, Davis A, Duff J, Dugas MA, Duncan A, Evans B, Feldman J, Felmet K, Fisher G, Frankel L, Jeffries H, Greenwald B, Gutierrez J, Hall M, Han YY, Hanson J, Hazelzet J, Hernan L, Kiff J, Kissoon N, Kon A, Irazuzta J, Lin J, Lorts A, Mariscalco M, Mehta R, Nadel S, Nguyen T, Nicholson C, Peters M, Okhuysen-Cawley R, Poulton T, Relves M, Rodriguez A, Rozenfeld R, Schnitzler E, Shanley T, Kache S, Skippen P, Torres A, von Dessauer B, Weingarten J, Yeh T, Zaritsky A, Stojadinovic B, Zimmerman J, Zuckerberg A (2009) Clinical practice parameters for hemodynamic support of pediatric and neonatal septic shock: 2007 update from the American College of Critical Care Medicine. Crit Care Med 37(2):666-688. Erratum in: Crit Care Med. 2009 Apr;37(4):1536. Skache, Sara (corrected to Kache, Saraswati); Irazusta, Jose (corrected to Irazuzta, Jose)

Carcillo JA (2003) Pediatric septic shock and multiple organ failure. Crit Care Clin 19(3):413-440, viii. Review

Carcillo JA, Tasker RC (2006) Fluid resuscitation of hypovolemic shock: acute medicine's great triumph for children. Intensive Care Med 32(7):958-961, Epub 2006 May

de Oliveira CF, de Oliveira DS, Gottschald AF, Moura JD, Costa GA, Ventura AC, Fernandes JC, Vaz FA, Carcillo JA, Rivers EP, 
Troster EJ (2008) ACCM/PALS haemodynamic support guidelines for paediatric septic shock: an outcomes comparison with and without monitoring central venous oxygen saturation. Intensive Care Med 34(6):1065-1075, Epub 2008 Mar 28

Goldstein B, Giroir B, Randolph A (2005) International Consensus Conference on Pediatric Sepsis. International pediatric sepsis consensus conference: definitions for sepsis and organ dysfunction in pediatrics. Pediatr Crit Care Med 6(1):2-8, Review

Han YY, Carcillo JA, Dragotta MA, Bills DM, Watson RS, Westerman ME, Orr RA (2003) Early reversal of pediatric-neonatal septic shock by community physicians is associated with improved outcome. Pediatrics 112(4):793-799, Review

Moloney-Harmon PA (2005) Pediatric sepsis: the infection unto death. Crit Care Nurs Clin North Am 17(4):417-429, xi
Oliveira CF, de Sá FR Nogueira, Oliveira DS, Gottschald AF, Moura JD, Shibata AR, Troster EJ, Vaz FA, Carcillo JA (2008) Time- and fluidsensitive resuscitation for hemodynamic support of children in septic shock: barriers to the implementation of the American college of critical care medicine/pediatric advanced life support guidelines in a pediatric intensive care unit in a developing world. Pediatr Emerg Care 24(12):810-815

Pizarro CF, Troster EJ, Damiani D, Carcillo JA (2005) Absolute and relative adrenal insufficiency in children with septic shock. Crit Care Med 33(4):855-859

Watson RS, Carcillo JA, Linde-Zwirble WT, Clermont G, Lidicker J, Angus DC (2003) The epidemiology of severe sepsis in children in the United States. Am J Respir Crit Care Med 167(5):695-701, Epub $2002 \mathrm{Nov}$ 
